\title{
Multipotent male germline stem cells (mGSCs) from neonate porcine testis
}

\author{
Zhiwei Niu ${ }^{1}$, Siyu Wu ${ }^{1}$, Chongyang $\mathrm{Wu}^{1}$, Na $\mathrm{Li}^{1}$, Haijing $\mathrm{Zhu}^{2}$, Weishuai Liu ${ }^{3}$, Jinlian \\ Hua $^{1^{*}}$ \\ ${ }^{1}$ College of Veterinary Medicine, Shaanxi Centre of Stem Cells Engineering \& Technology, Key Lab for Animal \\ Biotechnology of Agriculture Ministry of China, ${ }^{2}$ Northwest A\&F University, Yangling, Shaanxi, China; ${ }^{3}$ College of \\ Life Science, Yulin College, Yunlin, Shaanxi, PR China, Northwest A\&F University, Yangling, Shaanxi, China
}

\begin{abstract}
Spermatogonial stem cells (SSCs) are the foundation of spermatogenesis, during which unlimited spermatozoa is produced daily derived from SSCs in the testis throughout life of the male. Germline stem (GS) cells can be isolated from spermatogonia, which shared the characteristics of SSCs and embryonic stem cells (ESCs), and can be passaged stably in vitro. The study of GS cells contributes to understanding spermatogenesis process. However, little is known about the GS cells in domestic animals. Here, we report the successful establishment of a serum-and feeder-free system for multipotent male GS cells (mGSCs) from postnatal porcine testis. These cells expressed pluripotent markers, such as Oct4, Nanog, C-myc, and germline-specific markers including Vasa, CD90, CD49f, Gfral, Plzf and Dazl. Then we assayed the developmental potential of these cells in vitro. The porcine multipotent male germline stem cells (pmGSCs) can form embryoid bodies (EBs) by suspension culture. Immunofluorescence analysis showed that the EBs differentiated into neuron-specific enolase (NSE, ectoderm), $\alpha$-actin (mesoderm), and Pdxl (endoderm) positive cells. These cells induced by $10^{-6} \mathrm{M}$ retinoic acid $(R A)$ could be differentiated into spermatid-like cells which were positive for Acrosin. The pmGSCs has been cultured over 14 passages. Thus, we have established a long-term culture system for pmGSCs. This culture system provides a platform for the study of porcine $m G S C$ s.
\end{abstract}

Key words: Spermatogonial stem cells (SSCs), multipotent male GS cells (mGSCs), spermatid-like cells, porcine

\footnotetext{
*Author for correspondence: jinlianhua@nwsuaf.edu.cn
} 


\section{INTRODUCTION}

Spermatogonial stem cells (SSCs), also named as postnatal male germline stem cells (mGSCs), are the foundation of spermatogenesis, during which unlimited spermatozoa is produced daily derived from SSCs in the testis throughout life of the male. The previous studies demonstrated that long-term cultured mouse germline stem cells (GSCs) were derived from gonocytes and spermatogonia in appropriate microenvironments, and these cells can be passaged for more than two years without losing fertility (Kanatsu-Shinohara et al. 2005). Additionally, mGSCs were obtained from mouse testes, and they are morphologically similar to embryonic stem cells (ESCs) and contribute to embryonic development following microinjection into blastocysts (Oatley et al. 2006; Dym et al. 2009; He et al. 2010).

A long or short term culture system for mGSCs has been established for human, mouse, and other mammals (Akhondi et al. 2013; Guo et al. 2014). The cultured mGSCs also express the markers of SSCs, and differentiated into spermatozoa. Porcine is a convenient domestic species for current biological investigation and application, because it has diversified products of commercial value and relatively short gestation period ( $\mathrm{Lv}$ et al. 2012). Porcine is viewed as the most promising model for human being, because it has the similar metabolism, and their physiology are close to human beings (Cao et al. 2011; Lv et al. 2012). In mammalian species other than mouse and human, where ample attempts to generate ESC lines had limited success, GSC based gene targeting is a promising technique for the production of transgenic offspring.
Gene targeting would particularly mean a highly valuable tool in pigs because of their importance in agriculture and the organ sizes (Kuijk et al. 2009). However, there lack long-term culture system for pmGSCs, which restrict the progress of related studies using pmGSCs.

In this study, we have successfully obtained the establishment of a long-term culture system for multipotent male germline stem cells from postnatal porcine testis. This provides a promising platform for the study of mGSCs development.

\section{MATERIAL AND METHODS}

\section{Isolation and culture of male germline stem cells (mGSCs) from postnatal porcine testis}

Postnatal porcine testis (derived from Chinese Bamei) at the age of one day to three day were collected and delivered back to the laboratory on ice. All procedures were approved by Shaanxi Centre of Stem Cells Engineering \& Technology, Northwest A\&F University. The porcine were killed by standard methods and porcine testicular cells were isolated in our laboratory as previous report (Zhu et al. 2012), using CDD mixed digestive enzyme: $2.0 \mathrm{mg} / \mathrm{mL}$ collagenase IV (Invitrogen) $+20 \mu \mathrm{g} / \mathrm{mL}$ DNase (Roche) $+2.0 \mathrm{mg} / \mathrm{mL}$ dispase (Invitrogen) The dissociated cells were suspended in DMEM (Invitrogen) containing 10\% FBS (Hyclone, Logan, UT), $2.0 \mathrm{mM}$ Lglutamine (Invitrogen), 1\% nonessential amino acids (Invitrogen), $0.1 \mathrm{mM} \beta$ mercaptoethanol (Sigma), 100 unit $/ \mathrm{mL}$ penicillin and $100 \mathrm{mg} / \mathrm{mL}$ streptomycin (Invitrogen). The cells $\left(2 \times 10^{5}\right.$ cells/L were cultured in $35-\mathrm{mm}$ dishes coated with $0.1 \%$ gelatin (Sigma, St. Louis, MO) for $12 \mathrm{~h}$ at $37^{\circ} \mathrm{C}$ and $5 \% \mathrm{CO}_{2}$ for the cells 
to adhere (Hua et al. 2011). The noadhering cells were transferred onto new laminin-coated plates to culture in StemPro-34 containing $1 \% \quad \mathrm{~N} 2$ (Invitrogen), supplemented with 2\% B27 (Invitrogen), $2 \mathrm{mg} / \mathrm{mL}$ Bovine Serum Albumin (BSA, Sigma), $2 \mathrm{mM}$ Lglutamine (Invitrogen), 1\% non- essential amino acids (Invitrogen), $0.1 \mathrm{mM} \beta$ mercaptoethanol (Sigma), $20 \mathrm{ng} / \mathrm{mL}$ GDNF (glial cell line-derived neurotrophic factor, Peprotech, NJ, USA), and $2 \mathrm{ng} / \mathrm{mL}$ bFGF (basic fibroblast growth factor, Millipore, Billerica, MA, USA), 10 ng/mL EGF (epidermal growth factor, Millipore), $50 \quad \mathrm{ng} / \mathrm{mL} \quad$ GFRA1 (GDNF family receptor alpha-1, Sino Biological Inc.). Media was changed every second day and the cells were subcultured into new plates by $1 \times$ accutase (Millipore) for 5-10 min to dissociation the cells every 45 days.

\section{Growth curve}

PmGSCs (passage12) were seeded to 24well plates at a density of $2 \times 10^{4}$ cells/well. The proliferation ability was assessed by growth curve at an interval of $24 \mathrm{~h}$ (Cao et al. 2011). The cells were trypsinized and the cell number was determined every 2 days for eight days $(n=3)$. Cell population doubling times (PDT) was calculated from the formula: $\mathrm{PDT}=[\log (2) /(\log \mathrm{Nt}-\log \mathrm{N} 0)] \times($ time $\mathrm{x}-$ start time).

\section{Cell cycle analysis}

For cell cycle analysis, pmGSCs were cultured for $48 \mathrm{~h}$ and then suspended the cells into single cells and fixed them in $70 \%$ ice-cold ethanol for $30 \mathrm{~min}$. After that, cells were incubated by propidium iodide (PI, Sigma, USA) solution and RNase H (Beyotime, China) for $20 \mathrm{~min}$. Cell cycle analysis was determined by flow cytometry as previously reported (Cao et al. 2012; Sun et al. 2013).

\section{Alkaline phosphatase staining}

Alkaline phosphatase (AP) activity was determined essentially as described by previous study (Piedrahita et al. 1998; Niu et al. 2013).

\section{Immunofluorescence Staining}

The pmGSCs colonies were fixed with $4 \%$ PFA for $10 \mathrm{~min}$ at room temperature, followed by three washes in cold PBS for $5 \mathrm{~min}$. Then cells were treated with $0.1 \%$ Triton X-100 for $10 \mathrm{~min}$ at room temperature and blocked by blocking solution (PBS $+1 \%$ BSA) for a minimum of $30 \mathrm{~min}$ before being washed with PBS and stained with primary antibodies including Oct-4 (1:500, Chemicon), Sox2 (1:200, Chemicon), Nanog (1:200; Chemicon), C-kit (1:50, Biolegend), Cmyc (1:500, Chemicon), GFR $\alpha 1$ (1:100, Santa Cruz), Plzf (1:200, Santa Cruz), CD90 (1:100, BD). The appropriate FITCconjugated secondary antibodies were used according to the manufacturer's manual (1:500, Chemicon). The nuclei of induced cells were stained by $5 \mu \mathrm{g} / \mathrm{mL}$ Hoechst33342 (Sigma) for $5 \mathrm{~min}$. At the same time, the negative controls were stained with secondary antibodies: goat anti-rabbit $\mathrm{IgG}$ and goat anti-mouse IgG.

The induced cultures were analyzed by using antibodies against Stra8 (1:100, Abcam), Scp3 (1:200, Santa Cruz, USA), Acrosin $\quad(1: 100, \quad$ Santa Cruz Biotechnology, Inc., CA), neuron-specifc enolase (NSE, 1:100, Beijing Zhongshan Golden Bridge Biochemical Factory), mouse anti-human cardiac $\alpha$-actin (1:500, Sigma) and Pdx1 (1:200, Abcam, Cambridge, MA, USA).

$R T-P C R$

Total RNA for RT-PCR analysis were extracted from typical pmGSCs or EBs using TRIzol (QIANGEN). The cDNA 
was synthesized based on 500 ng RNA with a commercially available kit (TaKaRa, Biotech. Co. Ltd.). The PCR steps included denaturation at $94^{\circ} \mathrm{C}$ for 5 min, followed by repeated cycles $30 \mathrm{sec}$ at $95^{\circ} \mathrm{C}, 54^{\circ} \mathrm{C}-58^{\circ} \mathrm{C}$ for $30 \mathrm{sec}, 72^{\circ} \mathrm{C}$ for $30-60$ sec, 35 cycles and final extension at $72^{\circ} \mathrm{C}$ for $10 \mathrm{~min}$. The primers were designed based on the sequences of the open reading frame from the NCBI GenBank and synthesized by AuGCT Biotechnology (Beijing). The PCR primers and the length of the amplified products are shown in Table 1. The PCR products were analyzed in $1.5 \%$ agarose (Invitrogen) gel electrophoresis, stained with ethidium bromide (Invitrogen), and visualized under UV illumination (Hu et al. 2012).

Table 1- The primer sequences

\begin{tabular}{llll}
\hline Gene & Forward Primer $\left(5^{\prime} \rightarrow 3^{\prime}\right)$ & Reverse Primer $\left(5^{\prime} \rightarrow 3^{\prime}\right)$ & Products \\
\hline Oct4 & gctgacaacaacgagaatc & ttgcgaatagtcactgctt & 252 \\
Sox2 & gcccaggagaacccaagat & gggtgccetgctgcgagta & 520 \\
C-Myc & ctggtgggcgagatcatca & cactgccatgaatgatgttcc & 304 \\
Nanog & gcgaatcttcaccaatg & tttctgccacctcttac & 407 \\
Klf4 & tgtcatcctgccctgccg & cggtagtgcctggtcagttcat & 455 \\
Vasa & Agaggacgaggtggttac & gcgatgttgttattcagtgt & 296 \\
CD90 & tacgacatcaaggtcctctac & cacgaggtgttctggattag & 176 \\
CD49f, & gattctatgtttggcgttgc & aaagatggcgatttaccc & 176 \\
Gfra1 & ctcgcctactctgggcttat & caggtcgtttccactgttgc & 108 \\
Dazl & atgttaggatggatgaaactgagatta & ccatggaaatttatctgtfattctact & 178 \\
$\beta$-Actin & gcggcatccacgaaactac & tgatctccttctgcatcctgtc & 138 \\
\hline
\end{tabular}

\section{Characterization and in vitro differentiation of pmGSCs}

The pmGSCs were digested and plated on an uncoated cell culture suspension dish (Corning) at $3 \times 10^{5}$ cells per $3.5 \mathrm{~cm}$ dish. After three days of culture, the cells aggregated and formed 'embryoid bodies' (EBs). The resulting EBs, whose diameters were approximately $100 \mu \mathrm{m}$, and transferred in a 48-well culture plate (1015 EBs per well) coated with $0.1 \%$ gelatin (Niu et al. 2013) to investigate the potentiality of spontaneous differentiation. These EBs were attached for 3-20 days and then were analyzed by immunofluorescence staining and RTPCR. Some EBs were plated in the previous induced medium (Hua et al. 2011). In order to investigate the spermatozoa differentiation potential, EBs were dissociated into small clusters or single cells and transferred into the same differentiated medium consisting of $10^{-6}$ $M$ retinoic acid (RA) for 7-14 days, without cytokines to induce differentiation (Nayernia et al. 2006; Hua et al. 2011).

\section{RESULTS}

\section{Morphologic characteristics of the pmGSCs}

The typical primary testicular cells isolated from porcine testis tissue were large and round. They displayed big spherical nucleus and high nuclear-tocytoplasm ratio. The diameter of these cells was 10 to $20 \mu \mathrm{m}$ (Fig. 1A). After 36$48 \mathrm{~h}$, the spermatogonia proliferated and aggregated into colony (Fig. 1, red arrow). Around the colony, there were Somatic cells (Fig. 1B, blue arrow). During passage 1 to passage 4, they were ES-like colonies (Fig. 1C). At passage 5, some cells were fibroblast-like or epithelioid-like cells 
(Fig. 1D). Between passage 5 to passage 10 , some pmGSCs maintained fibroblastlike, other became ES-like colonies (Fig. 1E). In our study, the typical pmGSCs derived from neonate testis were

P0-12h

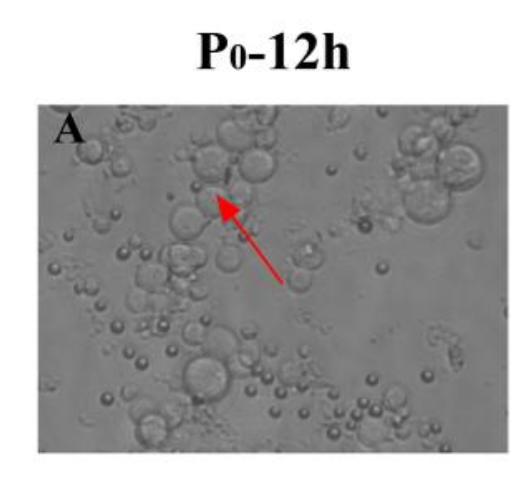

P5

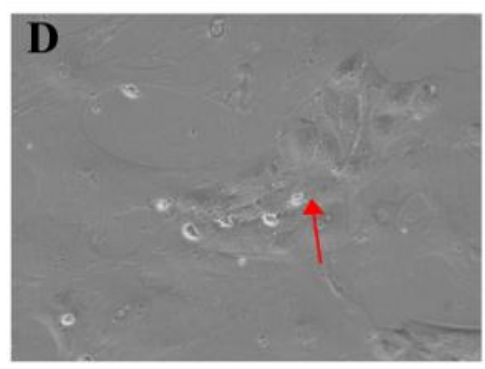

subcultured up to $14^{\text {th }}$ passage. After passage 14, all of pmGSCs displayed as fibroblast-like morphology (Fig. 1F).
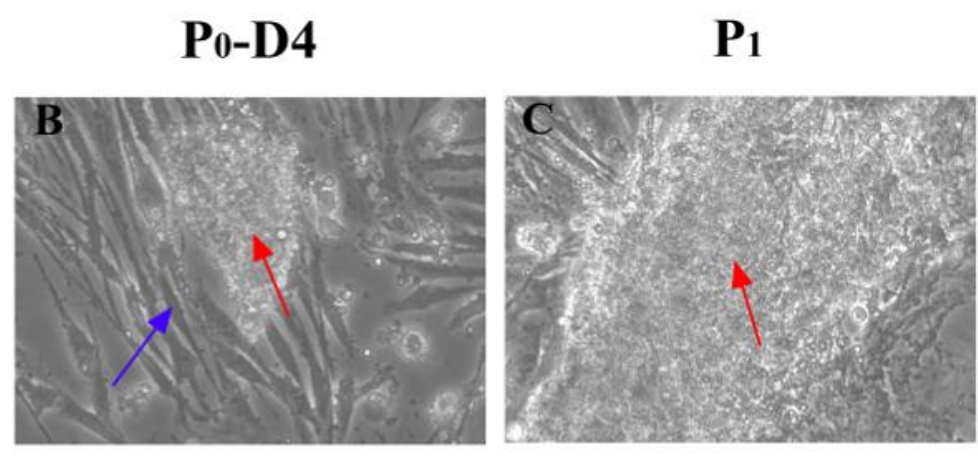

P10

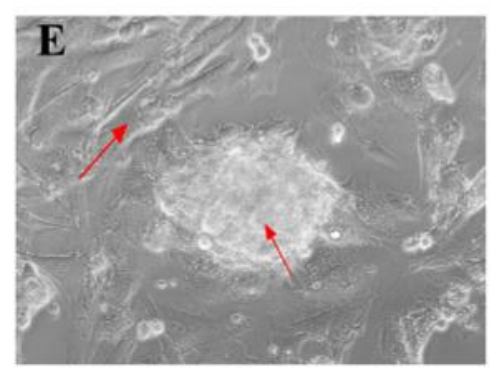

\section{$\mathbf{P}_{15}$}

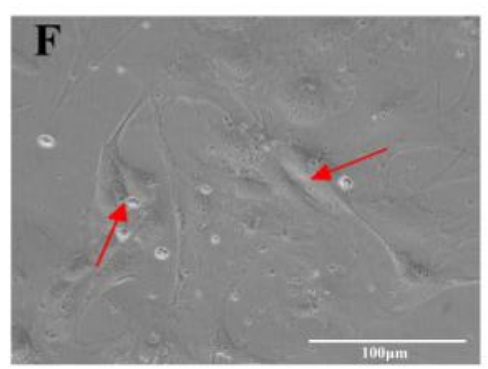

Figure 1- Morphology of pmGSCs. A, Dispersed cells were shown at $12 \mathrm{~h}$ after culture. B, pmGSCs colonies (red arrow). Somatic cells around the colonies (blue arrow). C, pmGSC colonies became bigger. D, pmGSC cell became fibroblast-like. E, Some pmGSCs exhibited as ES-like colony, but some pmGSCs were fibroblast-like (two red arrow, both indicate pmGSCs). F, After passage 15, most of pmGSCs exhibited as fibroblast-like. Scale bar $=100 \mu \mathrm{m}$.

\section{Characterization of pmGSCs}

Immunofluorescence analysis showed that isolated pmGSCs at passage 2 were positive for pluripotent markers: Oct4, Sox2, Nanog, C-myc and germlinespecific marker Gfral (Fig. 2B), and pmGSCs colonies were positive for AP staining (Fig. 2B). RT-PCR analysis of pmGSCs showed these cells expressed pluripotent marker: Oct4, Nanog, Klf4, Cmyc and germline-specific marker: Vasa,
CD90, CD49f, Gfra1, and Dazl (Fig. 2C). In addition, these cells were positive for germline-specific marker: CD90, Gfra1, Plzf analysed by immunofluorescence assay (Fig. 3A). RT-PCR analysis of pmGSCs at passage 8 showed these cells still expressed Oct4, Nanog, C-myc, Vasa, CD90, CD49f, Gfra1, and Dazl (Fig. 3B). 

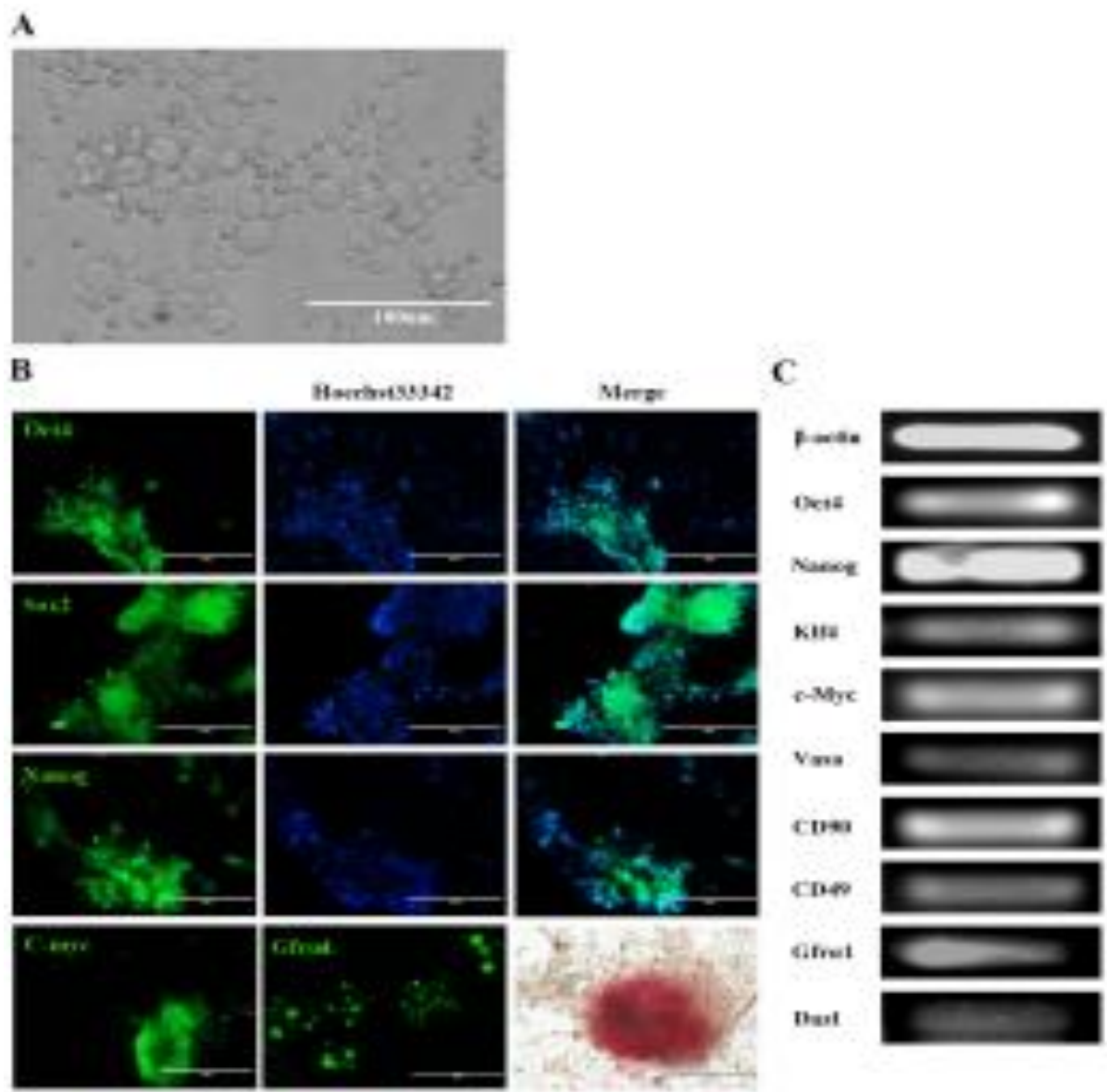

nan

Figure 2- Characterization of testicular cells isolated from testicular tissue. A,The morphology of the isolated pmGSCs. Scale bar $=100 \mu \mathrm{m}$. B, Immunofluorescence analysis showed that isolated pmGSCs were positive for Oct4, Sox2, Nanog, C-myc and Gfra1. pmGSCs colonies were positive for AP staining (red). Scale bar $=200 \mu \mathrm{m}$.

C, RT-PCR analysis showed pmGSCs expressed Oct4, Nanog, Klf4, C-myc, Vasa, CD90, CD49f, Gfra1 and Dazl. 


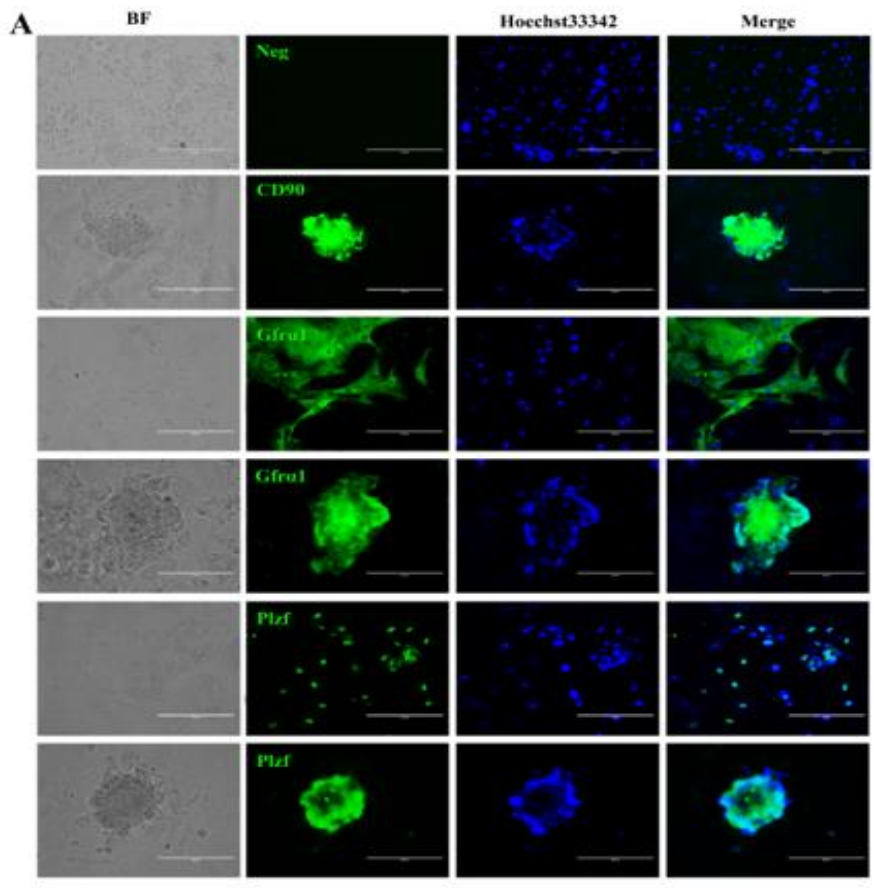

B

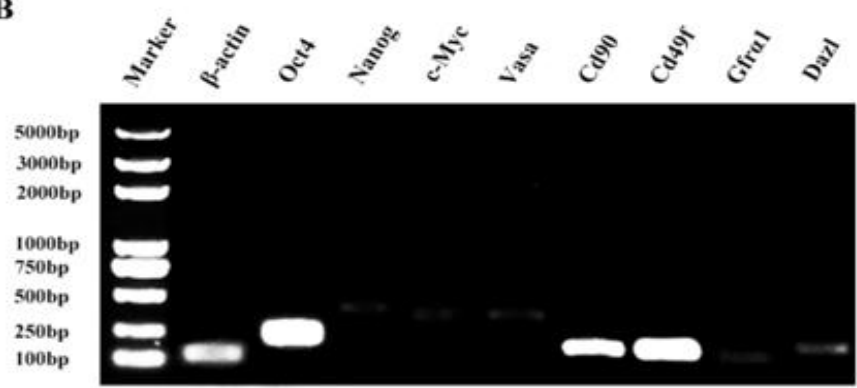

Figure 3- Characterization of pmGSCs at passage 10 (Immunofluorescence analysis) and passage 8 (RT-PCR). A, Immunofluorescence analysis showed that pmGSCs at passage 10 were positive for germline-specific marker: CD90, Gfra1, Plzf. B, RT-PCR analysis of pmGSCs at passage 8 showed these cells expressed Oct4, Nanog, C-myc, Vasa, CD90, CD49f, Gfra1 and Dazl. Scale bar=200 $\mu \mathrm{m}$.

Growth curve and cell cycle of pmGSCs Growth curve has been drawn based on the cell number for 8 days, and the cells were dissociated and counted every 2 days (Fig. 4A). The population doubling time of these cells (passage 12) was $50.2 \mathrm{~h}$. The cells cycle were detected, and G1 phase cells accounted up to $81.231 \%$ (Fig. 4B). 


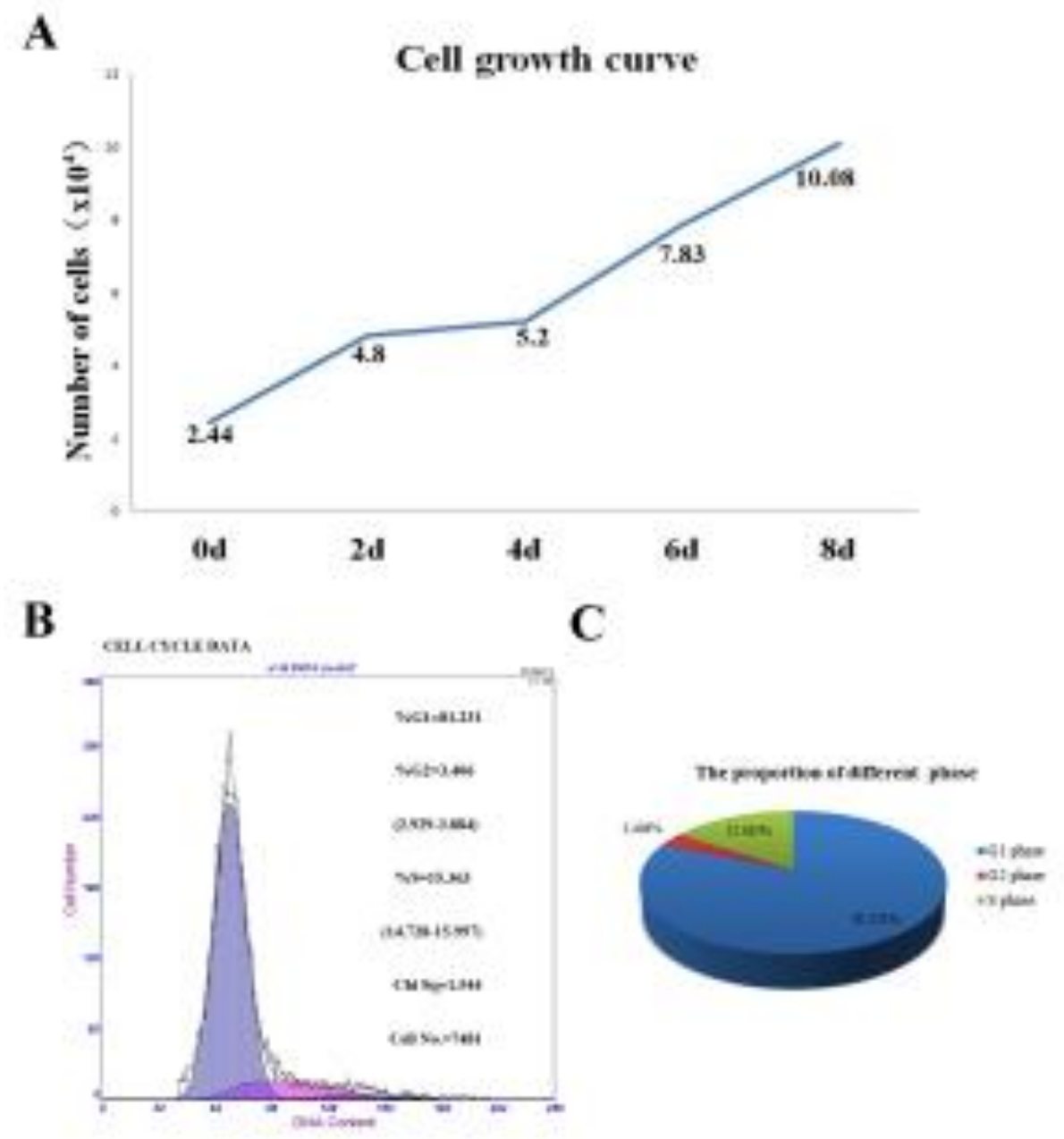

Figure 4- Growth curve and growth cycle of pmGSCs. A, Growth curve of pmGSCs. B, Cell cycle of pmGSCs.

\section{Differentiation potential of pmGSCs}

We transferred the $3^{\text {rd }}$ passage pmGSCs into suspension culture system. After 5 days, embryoid bodies (EBs) have been formed spontaneously. Then we collected the EBs and transferred them into adherent culture system. EBs attached to the plastic plates after 3 days. Peripheral cells of EBs have spread and formed cellular monolayers (Fig. 5A). The specific markers of germline and pluripotent cells were not detected in these EBs by RTPCR assay (Fig. 5B), and three germ layers specific markers, such as NSE (ectoderm), $\alpha$-actin (mesoderm), and Pdx 1 (endoderm) were expressed (Fig. 5C).

After passage 10, the developmental capacity of these cultured pmGSCs in vitro was investigated, and the cells were induced by RA for 6 days. The induced cells were positive for Stra8 and Scp3 by immunofluorescence assay (Fig. 6A). A few of the induced cells exhibited as spermatid-like morphology, and were positive for Acrosin (Fig. 6B). 
$\mathbf{A}$

B
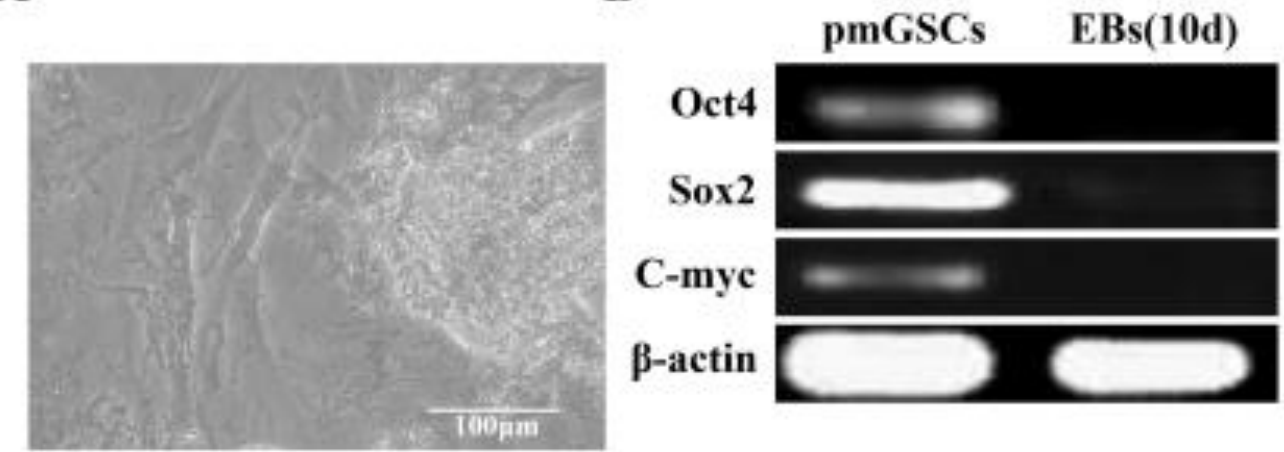

C
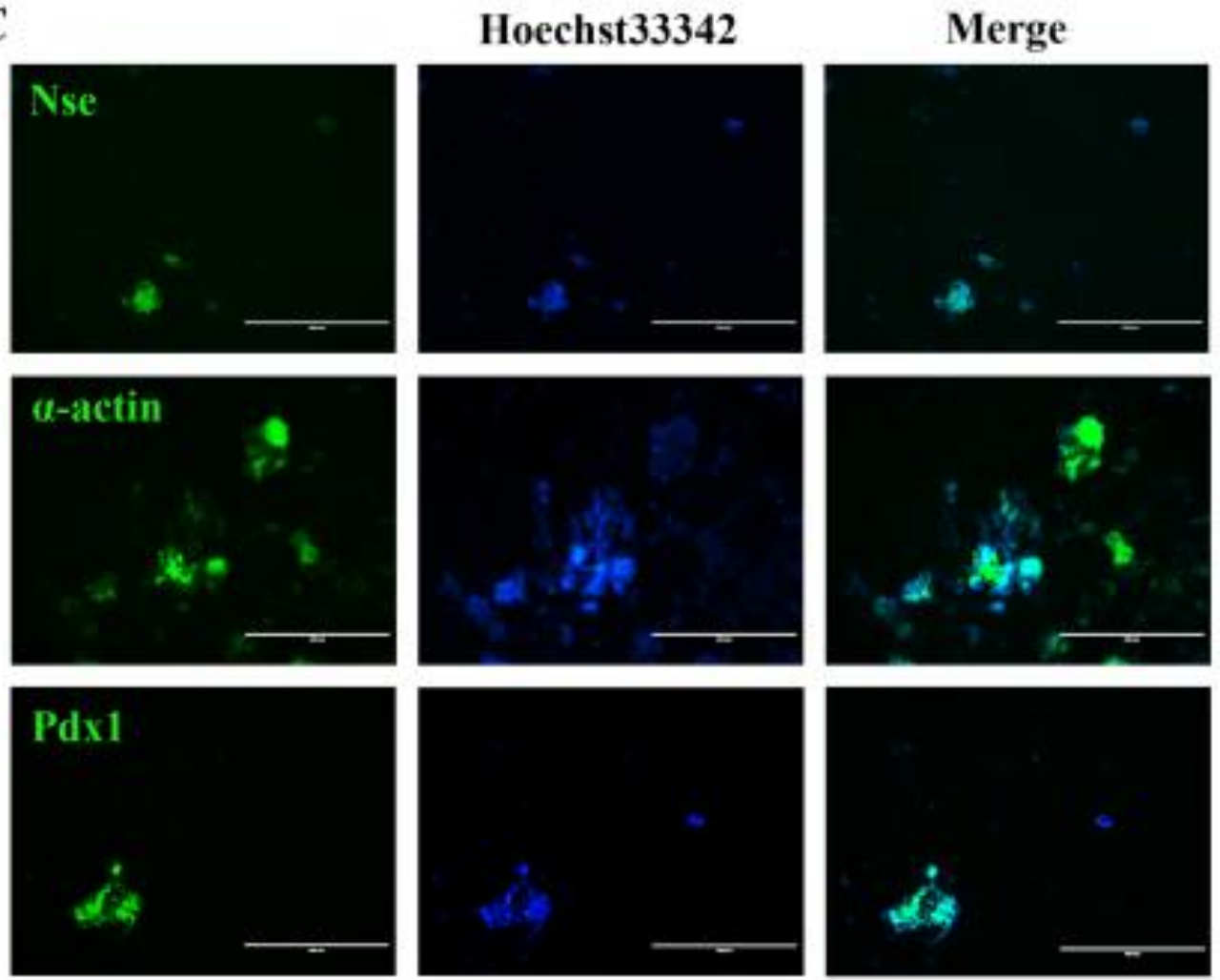

Figure 5- pmGSCs differentiated into three germ layer cell types. A, EBs derived from pmGSCs. Scale bar $=100 \mu \mathrm{m}$. B, RT-PCR analysis of EBs derived-pmGSCs showed that cells were negative for Oct4, Sox2, C-myc. C, Immunofluorescent staining assay showed that the EBs derived-pmGSCs expressed NSE, a-actin, Pdx1. Scale bar $=400 \mu \mathrm{m}$. 


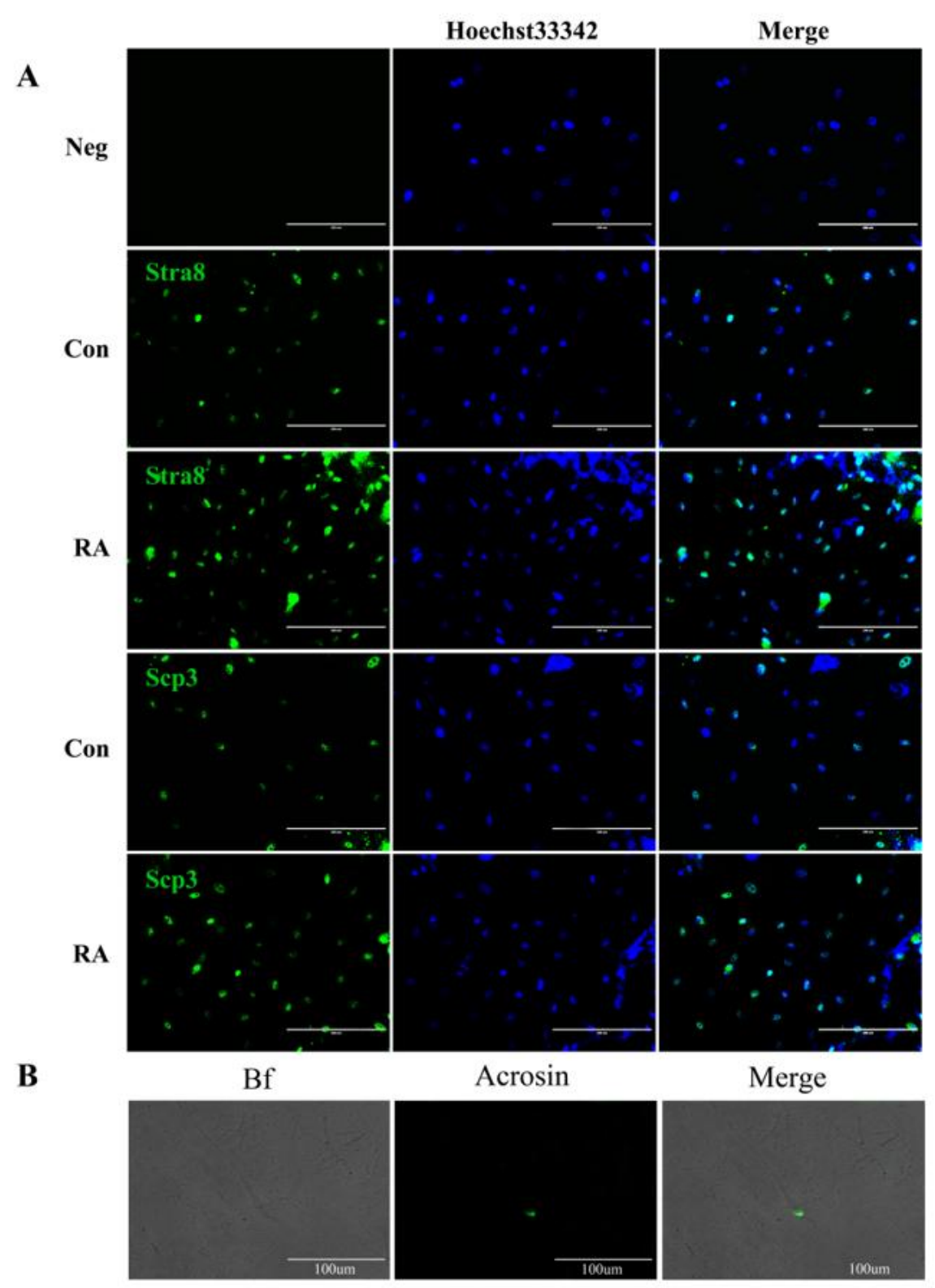

Figure 6- Spermatid-like cells were induced from pmGSCs. A, The cells induced by RA were positive for Stra8 and Scp3. Scale bar $=100 \mu \mathrm{m}$. B, The cells induced by RA displayed as spermatidlike, and were positive for Acrosin. Scale bar $=100 \mu \mathrm{m}$.

\section{DISCUSSION}

It has been known since 2004 that, under certain culture conditions, pluripotent/ multipotent ES-like cells can be established from germline stem cells derived from mouse testes (KanatsuShinohara et al. 2004; Kanatsu-Shinohara et al. 2008; Kanatsu-Shinohara et al. 2013). Previous studies demonstrated that several pluripotent/multipotent germline stem cells have been established and characterized by different research groups using various protocols, and murine and human male GSCs can be cultured for long periods and retain the capacity to differentiate into multiple cell types in the presence of serum or feeder cells in vitro. Recently, porcine, goat and bovine mGSCs were cultured and characterized, and these cells shared similar 
characteristics of ES cells and GSCs (Kanatsu-Shinohara et al. 2008; Goel et al. 2009; Kuijk et al. 2009; Hua et al. 2011; Zeng et al. 2013; Zheng et al. 2013; Park et al. 2014). However, the methods available for isolating SSCs and mGSCs from porcine testicular cells have a low efficiency of cell separating, and it is difficult to establish a stable livestock mGSCs line (Zeng et al. 2013; Zheng et al. 2013; Park et al. 2014). In this study, we used Laminin in combination with differentiating plating (DP) method to isolate SSCs from postnatal porcine testicular cells.

Niche and microenvironments play important roles in the fate and behavior of stem cells (Griswold et al. 2013; DeFalco et al. 2015). Recent studies demonstrate the importance of growth factors and extracellular matrix (ECM) on stem cell and germ cell behavior (Kuijk et al. 2009; Zhu et al. 2012). Derivation of pluripotent cells from male germ cells is enhanced by the cytokines, such as epidermal growth factor (EGF), Colony stimulating factor 1 (CSF1), Leukemia inhibitory factor (LIF), Glial cell-derived neurotrophic factor (GDNF) (Kuijk et al. 2009; Zhu et al. 2012). GDNF is secreted by the somatic Sertoli cells and peritubular cells, and is a well-known inducer of mouse SSC proliferation in vitro (Oatley et al. 2008). Binding of GDNF to the GDNF-family receptor $\alpha 1$ (GFR $\alpha 1)$ catalyses the activation of the c-RET receptor, which activates several signal cascades, such as PI3K/AKT, MEK and SCR kinases (Zheng et al. 2013). GDNF activation upregulates expression of the transcription factor-encoding genes Bcl6b, Etv5, and Lhx1, which influence mouse SSC selfrenewal (Oatley and Brinster 2008). EGF and FGF had a positive effect on the number and size of the SSC-like colonies in primary cultures of neonate pig testis. In vitro, propagation of porcine mGSCs could be maintained in the presence of $1 \%$ FBS and supplementation of growth factors for one month (Zheng et al. 2013). The authors evaluated the effects of growth factors based on the number of colonies of SSC-like, and expression level of SSC markers and suggest that FGF can impede successful derivation of porcine SSCs from neonate pig testis (Goel et al. 2009). Update, the effects of cytokines and growth factors on porcine mGSCs were not clear (Goel et al. 2009; Zheng et al. 2013). In the present study, the pmGSCs have been cultured up to 14 passages for over two months, while still maintained a typical morphology to mouse and human GSCs, indicating that our cultural system could simulate the cellular niche in seminiferous tubules that maintained the proliferation and differentiation of mGSCs in vitro. In our study, we also found that the efficiency of establishment of pmGSC line is greatly affected by the age of porcine. This is consistent with previously established in the mouse and dairy goat (Zhu et al. 2012). More important, the growth curve and cell cycle analysis demonstrated that porcine mGSCs cultured in the serum- and feeder- free system could be expanded and maintained stably in the presence of N2B27 and supplementation of growth factors for two month. However, it is unclear whether the cultured porcine mGSCs retain their full differentiation capacity of spermatogenesis after transplantation (Corad et al. 2014). This study preliminarily demonstrated our established serum- and feeder-free culture system were suitable for the establishment of porcine mGSCs line. 
It was reported that after in vitro cultures of mammalian mGSCs shared similar phenotypical characteristics as SSCs and pluripotent ES cells (Oatley and Brinster 2008; SuYoung et al. 2009; Hua et al. 2011). Pluripotency related markers of NANOG, Oct-4, and SSC markers: promyelocytic leukemia zinc-finger (PLZF), GFRA1, ITGA6 ( $\alpha 6$-integrin, CD49f), and THY1 were expressed at higher levels (Kanatsu-Shinohara et al. 2008; Kuijk et al. 2009; Zheng et al. 2013). Our cultured pmGSCs shared similar characteristics as pluripotent ES cells and SSCs (Kanatsu-Shinohara et al. 2008). They were positive for Oct 4 , Nanog, C-myc and germ cell specific marker Vasa, Gfra1, Plzf, CD49f, CD90. Additionally, the pmGSCs differentiated into EBs in suspension culture, and further differentiated into NSE (ectoderm), a-actin (mesoderm), and Pdx1 (endoderm) positive cells. The characteristics of these cells were consistent with mouse and human mGSCs (Guan et al. 2006; Seandel et al. 2007; Golestaneh et al. 2009; Kim et al. 2010). These results suggest that these cultured pmGSCs maintained the characteristics of pluripotent ES cells and SSCs.

In conclusion, we have successfully established in the serum- and feeder-free system for pmGSCs from postnatal porcine testis, and demonstrated these cells have characteristics similar to ESCs, and SSCs. This study will provide a new approach to establish porcine GSC lines.

\section{REFERENCES}

Akhondi MM, Mohazzab A, Jeddi-Tehrani M, Sadeghi MR, Eidi A, et al. Propagation of human germ stem cells in long-term culture. Iran $J$ Reprod Med. 2013;11(7):551-558.

Cao H, Chu Y, Lv X, Qiu P, Liu C, Zhang H, et al. GSK3 inhibitor-BIO regulates proliferation of immortalized pancreatic mesenchymal stem cells (iPMSCs). PloS one, 2012; 7: e31502.

Cao H, Chu Y, Zhu H, Sun J, Pu Y, Gao Z, et al. Characterization of immortalized mesenchymal stem cells derived 328 from foetal porcine pancreas. Cell Proliferation. 2011;44: 19-32.

Conrad S, Azizi H, Hatami M, Kubista M, Bonin M, Hennenlotter J, et al. Differential gene expression profiling of enriched human spermatogonia after short- and long-term culture. Biomed Res Int. 2014;138350.

DeFalco T, Potter SJ, Williams AV, Waller B, Kan MJ, Capel B. Macrophages contribute to the spermatogonial niche in the adult testis. Cell Rep. 2015; 12:11071119.

Dym M, Kokkinaki M, and $\mathrm{He} Z$. Spermatogonialstem cells: mouse and human comparisons. Birth Defects Research Part C: Embryo Today: Reviews 2009; 87: 27-34.

Goel S, Fujihara M, Tsuchiya K, Takagi Y, Minami N, Yamada M, et al. Multipotential ability of primitive germ cells from neonatal pig testis cultured in vitro. Reprod Fertility and Develop. 2009;21:696-708.

Golestaneh N, Kokkinaki M, Pant D, Jiang J, DeStefano D, Fernandez-Bueno C, et al. Pluripotent stem cells derived from adult human testes. Stem Cells Dev. 2009;18: 1115-1126.

Griswold M D and Oatley J M. Concise review: Defining characteristics of mammalian spermatogenic stem cells. Stem Cells. 2013;31: 8-11.

Guan K, Nayernia K, Maier L S, Wagner S, Dressel R, Lee J H, et al. Pluripotency of 
spermatogonial stem cells from adult mouse testis. Nature. 2006;440: 11991203.

Guo Y, Hai Y, Gong Y, Li Z, and He Z. Characterization, Isolation, and Culture of Mouse and Human Spermatogonial Stem Cells. JCel Physiol. 2014;229: 407-413.

He Z, Kokkinaki M, Jiang J, Dobrinski I, and Dym M. Isolation, characterization, and culture of human spermatogonia. Biol Reprod. 2010; 82: 363-372.

Hu Y, Sun J, Wang J, Wang L, Bai Y, Yu M, et al. Characterization of female germ-like cells derived from mouse embryonic stem cells through expression of GFP under the control of Figla promoter. J Cel Biochem. 2012;113: 1111-1121.

Hua JL, Zhu HJ, Pan SH, Liu C, Sun J W, Ma XL, et al. Pluripotent Male Germline Stem Cells from Goat Fetal Testis and Their Survival in Mouse Testis. Cel Reprogram.2011;13: 133-144.

Kanatsu-Shinohara M, Inoue K, Lee J, Yoshimoto M, Ogonuki N, Miki H, et al. Generation of pluripotent stem cells from neonatal mouse testis. Cell. 2004; 119: 1001-1012.

Kanatsu-Shinohara M, Ogonuki N, Iwano T, Lee J, Kazuki Y, Inoue K, et al. Genetic and epigenetic properties of mouse male germline stem cells during long-term culture. Development. 2005;132:41554163.

Kanatsu-Shinohara M, Lee J, Inoue K, Ogonuki N, Miki H, Toyokuni S, et al. Pluripotency of a single spermatogonial stem cell in mice. Biol Rreprod. 2008;78: 681-687.

Kanatsu-Shinohara M, and Shinohara T. Spermatogonial stem cell self-renewal and development. Annu Rev Cell Dev Biol. 2013;29; 163-187.

Kim HJ, Lee HJ, Lim JJ, Kwak KH, Kim JS, $\mathrm{Kim} \mathrm{JH}$, et al. Identification of an intermediate state as spermatogonial stem cells reprogram to multipotent cells. $\mathrm{Mol}$ Cells. 2010; 29: 519-526.

Kuijk EW, Colenbrander B, and Roelen BA. The effects of growth factors on in vitrocultured porcine testicular cells. Reprod. 2009;138: 721-731.

Lv X, Zhu H, Bai Y, Chu Z, Hu Y, Cao H, et al. Reversine promotes porcine muscle derived stem cells (PMDSCs) differentiation into female germ-like cells. J Cel Bioch.2012;113: 3629-3642.

Nayernia K, Nolte J, Michelmann HW, Lee JH, Rathsack K, Drusenheimer N, et al. In vitro-differentiated embryonic stem cells give rise to male gametes that can generate offspring mice. Devel Cell. 2006;11: 125 132.

Niu Z, Hu Y, Chu Z, Yu M, Bai Y, Wang L, et al. Germ-like cell differentiation from induced pluripotent stem cells(iPSCs). Cell Bioch Function. 2013;31: 12-19.

Oatley JM, and Brinster RL. Spermatogonialstem cells. Meth Enzymol 2006;419: 259-282.

Oatley JM, and Brinster RL. Regulation of spermatogonial stem cell self-renewal in mammals. Annu Rev Cell Dev Biol. 2008;24: 263-286.

Park MH, Park JE, Kim MS, Lee KY, Park HJ, Yun JI, et al. Development of a high-yield technique to isolate spermatogonial stem cells from porcine testes. J Assist Reprod Genet. 2014; 31:983-991.

Piedrahita JA, Moore K, Oetama B, Lee Ck, Scales N, Ramsoondar J, et al. Generation of transgenic porcine chimeras using primordial germ cell-derived colonies. Biol Rep. 1998;58: 1321-1329.

Seandel M, James D, Shmelkov SV, Falciatori I, Kim J, Chavala S, et al. Generation of functional multipotent adult stem cells from GPR125+germline progenitors. Nature.2007;449: 346-350. 
Sun J, Zhu H, Liu C, Li M, and Hua J. GDNF Up-Regulates c-Myc Transcription via the PI3K/Akt Pathway to Promote Dairy Goat Male Germline Stem Cells (mGSC) Proliferation. J Integ Agric. 2013;12: 10541065.

SuYoung $\mathrm{H}$ and Gupta M. Isolation and in vitro culture of pig spermatogonial stem cell. Asian-Australasian J Animal Sci. 2009; 22: 187-193.

Zeng W, Tang L, Bondareva A, Honaramooz A, Tanco V, Dores C, et al. Viral transduction of male germline stem cells results in transgene transmission after germ cell transplantation in pigs. Biol Reproduc. 2013;88: 27.
Zheng Y, He Y, An J, Qin J, Wang Y, Zhang $\mathrm{Y}$, et al. THY1 is a surface marker of porcine gonocytes. Reprod Fertil Develop. 2014; 26:533-539.

Zheng Y, Tian X, Zhang Y, Qin J, An J, and Zeng $\mathrm{W}$. In vitro propagation of male germline stem cells from piglets. $J$ Assisted Reprod Genetics 2013;30: 945-952.

Zhu H, Liu C, Li M, Sun J, Song W, and Hua J. Optimization of the conditions of isolation and culture of dairy goat male germline stem cells (mGSC). Animal Reprod Sci. 2012;137: 45-52.

Received: 17 Aug-2015 Accepted: 04 Oct- 2015 